\title{
Compressive Sensing based Gender Recognition
}

\author{
Suparna Biswas, Jaya Sil, Santi P. Maity
}

\begin{abstract}
This paper explores an integration of compressive sensing, curvelet transform, and Principal Component Analysis to develop a robust gender recognition method from face images. Compressive measurements of face images leading to a significant reduction in feature space. Here curvelet transform has been used to represent the face images with prominent edges, curvatures, boundaries and to offer sparse representation to apply compressive measurements on detailed subband. To extract the feature vector, Principal Component Analysis is applied on the reconstructed detailed subband. Performance of the proposed method is evaluated by employing different classifiers. The proposed method efficiently handles the effect of Gaussian noise maintaining high accuracy on gender recognition. Extensive experiments on FERET database, is conducted to substantiate our claim.
\end{abstract}

Keywords - Gender recognition, Curvelet transform, Compressive sensing, Principal Component Analysis.

\section{Introduction}

Gender recognition is an active area of research in computer vision and facing real life challenges for its commercialization. Gender recognition from face images using large set of training data has important real time applications in the fields of security, job distribution etc. It is known that men and women have discriminative facial features, which can be learned for gender recognition. In the present work we concentrate on developing an autonomous gender recognition system using face images by analyzing facial features.

Human perception involves observation of both coarse (global) and detailed (local) features of the face to identify and categorize a person. Currently different types of gender classification methods are available in the literature such as face based, gait based, hand based and many more. However, facial images are widely used for gender classification because facial images probably contain the most common biometric characteristic used by humans to make a personal recognition.

Dimensionality of features plays important role in classification accuracy because learning efficiency is affected due to presence of redundant and irrelevant features in training phase. Moreover, large number of features increases computational cost, slowing down the classification process and creates problem in real time

\section{Suparna Biswas}

Indian Institute of Engineering Science and Technology India

Jaya Sil

Indian Institute of Engineering Science and Technology India

Santi P. Maity

Indian Institute of Engineering Science and Technology India implementation. Therefore, appropriate feature extraction and feature selection methods together improve gender classification accuracy, which have been dealt in this paper proposing a comprehensive framework.

In 2006, Candes and Tao [1] proposed a new mathematical theory and algorithm of compressed sensing (CS) framework, which is a breakthrough of Nyquist sampling theorem. According to CS theory, the sampling frequency could be far below the Nyquist rate as long as signals are sparse and compressible in the measurement space. CS theory and sparse representation of signals allow processing with few numbers of samples and recovering it with low distortion. In this paper we present a CS theory based robust gender recognition scheme to solve the real time gender recognition problem.

Objective of this work is to extract the detail edge information utilizing curvelet transform (CT) which is more relevant to recognize the gender and also offers a sparse domain to reduce the storage space of input images. This paper actually proposes an integrated framework using CS, CT and Principal Component Analysis (PCA) for recognition of gender. PCA has been applied on reconstructed detail subband images to select important features with low dimensional space.

The paper is organized as follows: Section II presents a brief review of gender recognition method, Section III presents the scope and contributions of the proposed method. Section IV describes the proposed gender recognition method and in Section $\mathrm{V}$ results are discussed. Section VI concludes the paper.

\section{Literature Review}

Feature extraction methods for gender recognition can be broadly classified into two categories: geometric approaches and appearance based approaches. Dimension of feature vector increases when pixel intensity is used as features resulting increase of the computational time of recognition as well. Dimension reduction technique such as PCA [2], [3] provides a representation of image in reduced dimension space. Santana et al. [4] proposed a PCA based gender recognition method, where Support Vector Machine (SVM) is used for classification. Two dimensional PCA (2DPCA) is an extension of PCA, used for dimension reduction of feature vector. $\mathrm{Lu}$ et al. proposed a 2DPCA based expression invariant gender recognition method in [5]. Gender recognition includes Linear Discriminant analysis (LDA) [6] and Independent Componant Analysis (ICA) [7].

In 2002 Ojala et al. [8] introduced a new texture descriptor, Local Binary Pattern (LBP) for grayscale images. In [9] an LBP based feature extraction technique is proposed for recognition of gender. After dividing the face image into equal sized blocks, LBP is applied for multi-view gender classification. Alexandre [10] combined LBP with intensity and shape features using a multiscale fusion approach. In [11], LBP is combined with contrast information for gender classification where local contrast histograms are used as 
rotation invariant features. Mayo et al. [12] used statistical features such as mean, variance, skew and kurtosis together with LBP. Different enhancements inspired by LBP have been proposed to solve the problem of gender recognition [13],[14],[15],[16].

In order to recognize gender from a face image, it is important to extract all available gender related information from the image and analyzing the information which describes the image. Wavelet Transform (WT) [17] is an ideal tool to analyze the images, ensuring extraction of detail information from high frequency coefficients. CWT and SVM are used for classifying the gender [18], [19] from the facial images and compared with DWT, RADON along with SVM. In [19] Dyadic Wavelet Transform (DyWT) is used for face description. Here integrating DyWT and LBP, a new feature descriptor DyWT-LBP is proposed for gender recognition. DyWT is a kind of translation invariant WT which has a better potential for detection than DWT. Sun et al. [20] showed that feature selection is an important issue for gender classification. An appearance-based method for identifying the gender has been proposed in [21] using facial images on the basis of a pixel-pattern-based texture feature (PPBTF). In [22] two state-of-the-art texture descriptors, the LBP and Weber's law descriptor (WLD) are proposed for gender classification. For feature selection, the local Gabor binary pattern is combined with local discriminative analysis leads to significant improvement in classification.

Recently-emerged CS theory, which originally aims to address signal sensing and coding problems, has shown tremendous potential for other problems like pattern recognition. Chen et al. [23] presented a new technique for gender recognition utilizing the distributed compressive sensing theory and joint sparse model (JSM) for efficient coding of multiple inter-correlated signals. However, curse of dimensionality of feature space is another challenge to solve classification problem with minimum error rate. To solve the dimensionality issue we have presented a CS based gender recognition scheme.

\section{Scope and Contributions}

In most of the CS based recognition problem, CS has been used to improve the classifier performance. Different from the existing methods, the proposed technique, reduce the storage space of database and efficiently handle the gender recognition with high accuracy. The major contributions of the paper are as follows:

i) A new framework of gender recognition scheme is proposed, where CT plays a dual role, (a) extraction of detail edge information and (b) sparsification of input face images. ii) For faster implementation we use Smoothed Projected Landweber (SPL) for CS reconstruction.

iii) We propose a CS based gender recognition framework which improves recognition accuracy.

iv) We perform extensive experiments on publicly available face data set FERET. We computed the performance of the proposed method using, K-Nearest Neighbor (KNN), neural network (NN), Naive Bayes (NB) and Support Vector Machine (SVM) classifiers.

\section{Iv. Proposed Method}

In this section a CS based efficient gender detection framework has been proposed. The flow diagram of the proposed gender recognition scheme is shown in Fig. 1.

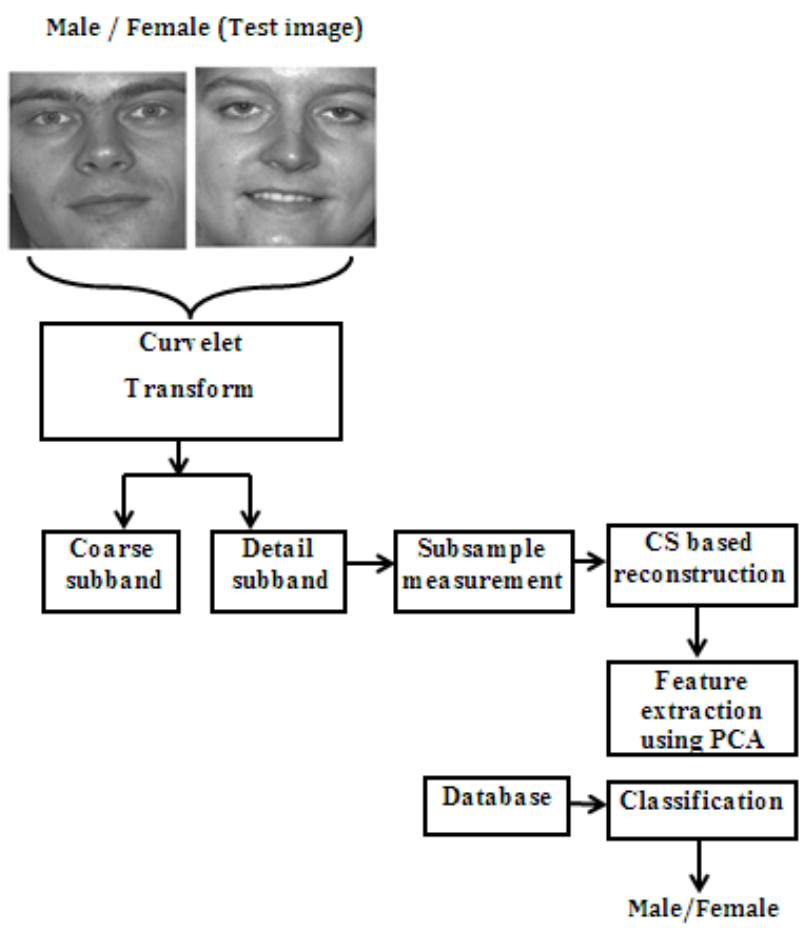

Fig. 1. Flow diagram of CS based gender recognition.

CT is a suitable basis function to sparsify the signal, because most of the coefficients are close to zero after signal transformation. Beside CT can capture better edge and directional information of image compare to other transforms. At the first step each face image (male and female) is decomposed using CT considering scale of 2 and angle 8 . Then extracting detail subband different percentage of samples (PS) are chosen and the subband images are reconstructed using CS based technique. For efficient reconstruction smoothed projected landweber (SPL) [24] method is used. Features are extracted in low dimensional space by applying PCA and different classifiers are used to detect the gender.

\section{v. Results and Discussions}

We evaluated the performance of the proposed algorithm on facial image database: FERET. The FERET [25] database contains frontal, left or right profile images and could have some variations in pose, expression and lightning. FERET database contains 11,338 images of 994 individuals and consists of different subsets such as frontal images (fa, fb) of different expressions, quarter left (ql), quarter right (qr), profile left $(\mathrm{pl})$, profile right (pr), half left (hl), half right (hr) and rotated images (ra, rb, rc).

In our experiments we use frontal, aligned and images of various pose and different expression (fa, fb, hl, hr subset images). During experiment we choose 600 images (mixed of fa, fb, hl, hr subset) among which 200 are used as test images. 
Table I demonstrates classification accuracy of FERET database for $\mathrm{PC}=50$ using $\mathrm{KNN}$ classifier. In KNN classifier we have considered three distance measures, which are euclidean, cityblock and cosine. From this table it is also noted that the maximum gender recognition accuracy is $97.78 \%$ for $\mathrm{PC}=50$ (for euclidean and city block distance measure). The recognition rate for other classifiers are shown in Table II, considering $\mathrm{PC}=50$ and $\mathrm{PS}=90 \%$. SVM classifier provides maximum recognition accuracy i.e. 98.33\% for RBF kernel function as shown in Table II. The ROC curve for SVM classifier is shown in Fig. 2.

TABLE I. GENDER RECOGNITION RATE OF FERET DATABASE USING KNN CLASSIFIER FOR PC=50

\begin{tabular}{|c|c|c|c|}
\hline PS & Euclidean & Cityblock & Cosine \\
\hline $10 \%$ & $77.78 \%$ & $77.78 \%$ & $77.78 \%$ \\
\hline $20 \%$ & $86.67 \%$ & $85.56 \%$ & $85.56 \%$ \\
\hline $30 \%$ & $92.22 \%$ & $93.33 \%$ & $93.33 \%$ \\
\hline $40 \%$ & $92.22 \%$ & $91.11 \%$ & $94.44 \%$ \\
\hline $50 \%$ & $94.44 \%$ & $95.56 \%$ & $95.56 \%$ \\
\hline $60 \%$ & $95.56 \%$ & $95.56 \%$ & $96.67 \%$ \\
\hline $70 \%$ & $97.78 \%$ & $95.56 \%$ & $96.67 \%$ \\
\hline $80 \%$ & $97.54 \%$ & $95.56 \%$ & $95.56 \%$ \\
\hline $90 \%$ & $97.78 \%$ & $97.78 \%$ & $96.67 \%$ \\
\hline
\end{tabular}

TABLE II. RECOGNITION RATE OF FERET DATABASE

\begin{tabular}{|c|c|}
\hline Classifier & $\begin{array}{c}\text { Recognition } \\
\text { rate in } \\
\text { percentage }\end{array}$ \\
\hline Naive Bayes & $94.77 \%$ \\
\hline Linear SVM & $96.44 \%$ \\
\hline Neural Network & $97.66 \%$ \\
\hline SVM with RBF & $98.33 \%$ \\
\hline Simple logistic & $95.88 \%$ \\
& \\
\hline
\end{tabular}

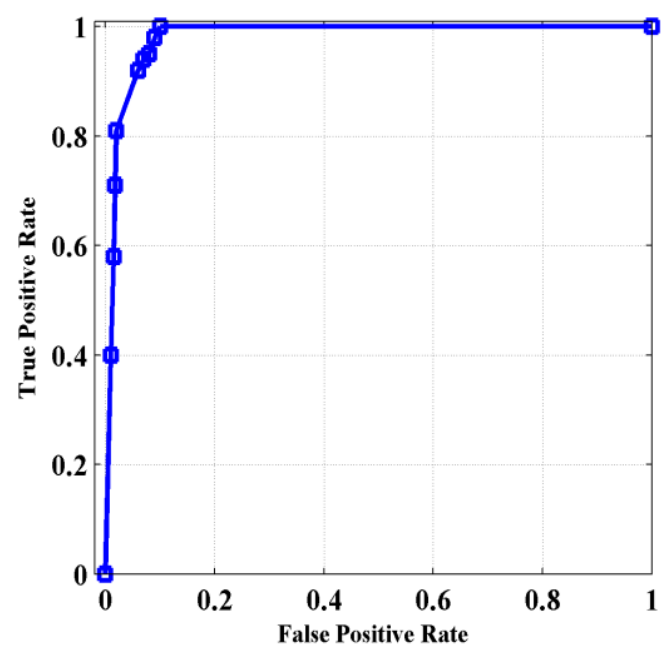

Fig. 2. ROC curve for SVM classifier
In order to observe the dependence of recognition rate on two parameters (feature dimension or PC value and PS value), we should trade-off the parameters and the graphical representation will become a three dimensional plot, such as shown in Figure 3 and Figure 4. Figure 3 and Figure 4 show how the recognition rate changes according to the changes of the feature dimension and PS value for KNN classifier (using city block distance). Figure 3 , shows that the best recognition rate $97.78 \%$ is obtained when the feature dimension is set to 50 and $\mathrm{PS}=70 \%$. In case of Fig. 4 the maximum recognition rate $97.78 \%$ is obtained for feature dimension equal to 50 and $\mathrm{PS}=90 \%$.

Recognition performance of the proposed method is studied for various degree of noise contamination on the face images. Gaussian noise is added with zero mean and varied variance. The classification results for different values of variance are presented in Table IV considering city block distance measure. We observe that the proposed method correctly classifies the noisy test images where accuracy has been varied by maximum $1 \%$.

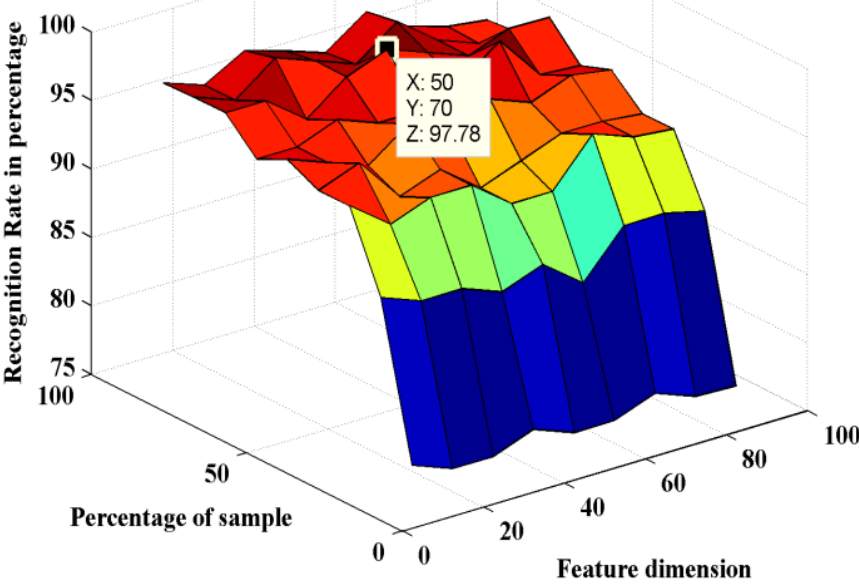

Fig. 3. Recognition Rate vs. feature dimension vs. PS for KNN classifier

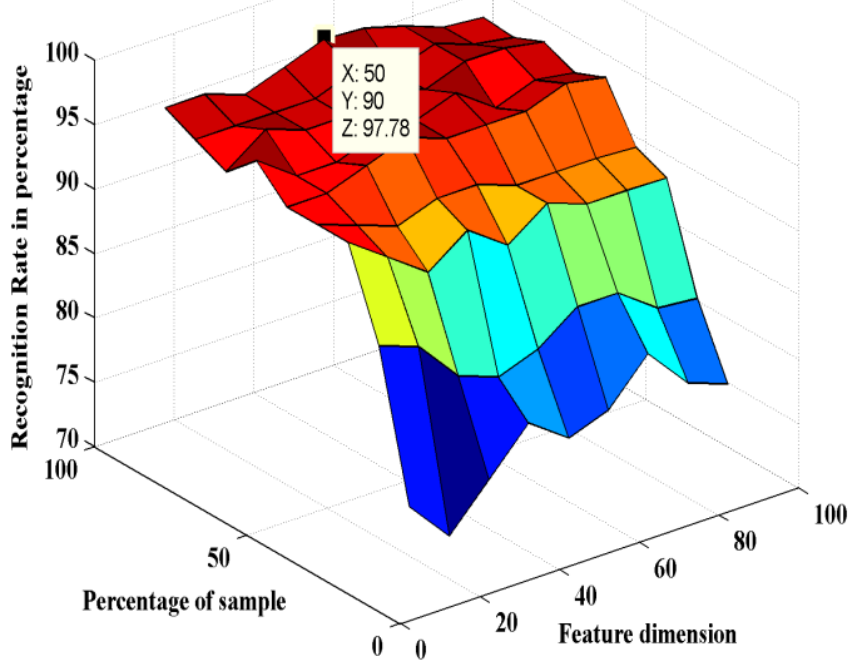

Fig. 4. Recognition Rate vs. feature dimension vs. PS for KNN classifier 
Proc. of the Sixth Intl. Conf. Advances in Computing, Communication and Information Technology- CCIT 2018

Copyright (C) Institute of Research Engineers and Doctors, USA. All rights reserved. ISBN: 978-1-63248-149-8 doi: 10.15224/978-1-63248-149-8-17

Comparisons with the existing methods are summarized in Table III. In Table III proposed method is compared with [26], [27] and [28]. The proposed method provide better recognition rate compared to [26], [27] and [28].

TABLE III. COMPARISON USING FERET DATABASE

\begin{tabular}{|c|c|}
\hline Method & $\begin{array}{c}\text { Maximum recognition } \\
\text { rate in percentage }\end{array}$ \\
\hline NN [26] & $90.07 \%$ \\
\hline SVM [26] & $84.44 \%$ \\
\hline LDA+Gabor wavelet [27] & $93.33 \%$ \\
\hline Wavelet+PCA[28] & $93 \%$ \\
\hline Proposed method & $97.78 \%$ (for KNN) \\
& $98.33 \%$ (for SVM) \\
\hline
\end{tabular}

TABLE IV. EFFECT OF NOISE ON FERET DATABASE (FOR $\mathrm{PS}=90 \%, \mathrm{PC}=50$ )

\begin{tabular}{|c|c|}
\hline Variance & $\begin{array}{c}\text { Average recognition } \\
\text { rate in percentage }\end{array}$ \\
\hline 0.01 & $97.75 \%$ \\
\hline 0.02 & $97.71 \%$ \\
\hline 0.1 & $97.26 \%$ \\
\hline 0.2 & $96.73 \%$ \\
\hline
\end{tabular}

\section{vi. Conclusions}

The paper proposed a facial feature based gender detection method in CS domain. The proposed integrated framework includes compact representation, dimensionality reduction and promising results while evaluation classification accuracy using different classifiers. If we store the database by randomly selecting percentage of samples from the detail subband, it saves storage space. Due to low dimension of feature, it can be used for real time applications. The experimental results on FERET data set clearly demonstrate that our method performs better compare to other methods. In the work, effect of PS on gender recognition is learned by varying the $\mathrm{PC}$ and obtained the maximum recognition rate $98.33 \%$ for $\mathrm{PC}=50$ and $\mathrm{PS}=90 \%$ (SVM with $\mathrm{RBF}$ ). The proposed method is robust under the effect of Gaussian noise.

\section{References}

[1] E. J. Candes and T. Tao, "Near-optimal signal recovery from random projections: Universal encoding strategies?" IEEE Transactions on Information Theory, vol. 52, no. 12, pp. 5406-5425, 2006.
[2] L. D. Golomb B and S. T, "Sexnet: a neural network identifies sex from human faces," Adv Neural Inf Process Syst, vol. 3, 1991

[3] E. B. O. A. Abdi H., Valentin D, "More about the difference between men and women: evidence from linear neural networks and the principal component approach," Perception, vol. 24, no. 5, pp. 539-562, May 1995.

[4] D. H.-S. M. Castrillon-Santana, J. Lorenzo-Navarro and J. IsernGonzalez, "Face description for perceptual user interfaces," in Proc. Conference of the Spanish Association for Artificial Intelligence, (CAEPIA), 2006, pp. 221-230.

[5] L. Lu and P. Shi, "A novel fusion-based method for expressioninvariant gender classification," in Proc. IEEE International Conference on Acoustics, Speech and Signal Processing.

[6] J. Bekios-Calfa, J. M. Buenaposada, and L. Baumela, "Revisiting linear discriminant techniques in gender recognition," IEEE Transactions on Pattern Analysis and Machine Intelligence, vol. 33, no. 4, pp. 858-864, 2011.

[7] H. Lu and H. Lin, "Gender recognition using adaboosted feature," in Proc. Third International Conference on Natural Computation (ICNC 2007), vol. 2, 2007, pp. 646-650.

[8] T. Ojala, M. Pietikainen, and T. Maenpaa, "Multiresolution gray-scale and rotation invariant texture classification with local binary patterns," IEEE Transactions on Pattern Analysis and Machine Intelligence, vol. 24, no. 7, pp. 971-987, 2002.

[9] H.-C. Lian and B.-L. Lu, Multi-view Gender Classification Using Local Binary Patterns and Support Vector Machines. Berlin, Heidelberg: Springer Berlin Heidelberg, 2006, pp. 202-209.

[10] L. A. Alexandre, "Gender recognition: A multiscale decision fusion approach," Pattern Recognition Letters, vol. 31, no. 11, pp. 1422-1427, August 2010

[11] J. Ylioinas, A. Hadid, and M. Pietikainen, "Combining contrast information and local binary patterns for gender classification," in Proc. 17th Scandinavian Conference on Image Analysis.

[12] M. Mayo and E. Zhang, "Improving face gender classification by adding deliberately misaligned faces to the training data," in Proc. $23^{\text {rd }}$ International Conference Image and Vision Computing New Zealand, 2008, pp. $1-5$.

[13] C. W. X. Fu, Guojun Dai and L. Zhang, "Centralized gabor gradient histogram for facial gender recognition," in Proc. Sixth International Conference on Natural Computation, vol. 4, 2010, pp. 2070-2074.

[14] J. Yang, Y. Jiao, N. Xiong, and D. Park, "Fast face gender recognition by using local ternary pattern and extreme learning machine," KSII Transactions on Internet and Information Systems, vol. 7, no. 7, pp. 1705-1720, July 2013.

[15] L. B. Zhou and W. Han, "Local gradient increasing pattern (lgip) for facial representation and gender recognition," in Proc. 9th International Conference on Image Analysis and Recognition, 2012.

[16] Y. Fang and Z. Wang, "Improving lbp features for gender classification," in Proc. International Conference on Wavelet Analysis and Pattern Recognition, vol. 1, 2008, pp. 373-377.

[17] A. F. Basha, G. Shaira, and B. Jahangeer, "Face gender image classification using various wavelet transform and support vector machine with various kernels," International Journal of Computer Science, vol. 9, no. 2 , pp. $150-157,2012$

[18] P. Rai and P. Khanna, "Gender classification using radon and wavelet transforms," in Proc. 5th International Conference on Industrial and Information Systems, 2010, pp. 448-451.

[19] I. Ullah, M. Hussain, G. Muhammad, G. B. Hatim Aboalsamh, and A. M. Mirza, "Gender recognition from face images with dyadic wavelet transform and local binary pattern," International Journal on Artificial Intelligence Tools, vol. 22, no. 6, pp. 409-419, 2013. 
[20] Z. Sun, G. Bebis, X. Yuan, and S. J. Louis, "Genetic feature subset selection for gender classification: a comparison study," in Proc. Sixth IEEE Workshop on Applications of Computer Vision(WACV 2002)

Proceedings, 2002, pp. 165-170.

[21] H. Lu, Y. Huang, Y. Chen, and D. Yang, "Automatic gender recognition based on pixel-pattern-based texture feature," vol. 3, pp. 109116,032008 .

[22] I. Ullah, H. Aboalsamh, M. Hussain, G. Muhammad, and G. Bebis, "Gender classification from facial images using texture descriptors," vol. 15, pp. 801-812, 092014.

[23] D. Y. Chen and P. C. Hsieh, "Face-based gender recognition using compressive sensing," in Proc. International Symposium on Intelligent Signal Processing and Communications Systems, Nov 2012, pp. 157- 161.

[24] S. Mun and J. E. Fowler, "Block compressed sensing of images using directional transforms," in Proc. IEEE International Conference on Image Processing (ICIP), Nov. 2009, pp. 3021-3024.

[25] "The feret face database," http://www.vision.caltech.edu/htmlfiles/archive.html.

[26] S. A. R. P. J. Phillips, Hyeonjoon Moon and P. J. Rauss, "An experimental comparison of gender classification methods," Pattern Recognition Letters, vol. 29, no. 10, pp. 1544-1556, 2008.

[27] X. M. Leng and Y. D. Wang, "Improving generalization for gender classification," in Proc. 15th IEEE International Conference on Image Processing, 2008, pp. 1656-1659.

[28] S. Biswas and J. Sil, "Gender recognition using fusion of spatial and temporal features," in Advanced Computing, Networking and InformaticsVolume 1, 2014, pp. 109-116. 